\title{
Videjugando: focalización atencional y rememoración en preadolescentes ¿Pueden atender procesos simultáneos y dejar espacio a la publicidad?*
}

\author{
Lizardo Vargas ${ }^{* *}$ \\ Recibido: Enero 15 - Aprobado: Marzo 15
}

\begin{abstract}
Resumen
El objeto del estudio es identificar, en preadolescentes, el grado de esfuerzo o posible inhibición en el proceso de evocación episódica y semántica que ocurre cuando su atención es focalizada debido a la interacción con un videojuego, e inferir si pueden atender y procesar la publicidad que se exhibe en estos. Se realizó un experimento cuantitativo para analizar dos grupos de individuos pertenecientes a la etapa de Operaciones Formales -estado de desarrollo cognitivo propuesto por el psicólogo Piaget por el que pasan las personas de determinada edad-. Se observaron efectos diferentes en la rememoración de los recuerdos episódicos y semánticos en cuanto al tiempo de evocación y número de palabras utilizadas para expresar el recuerdo. Sin embargo, no se observó una diferencia significativa en cuanto al grado de seguridad con que lo evocado era expuesto. Se concluyó que la focalización atencional por videojuegos no exige distintos niveles de esfuerzo cognitivo para procesar información en simultáneo con la rememoración semántica o episódica, por lo que la publicidad exhibida sí puede ser atendida.
\end{abstract}

Palabras clave: preadolescentes, videojuegos, atención, memoria, publicidad.

* Investigación financiada por la Facultad de Comunicación de la Universidad de Piura.

** Director de Posgrado de la Facultad de Comunicación de la Universidad de Piura (Perú). Dicta Estrategias Publicitarias y Psicología del Consumidor en esa facultad, así como Metodologías de Investigación Cualitativa en el programa de doctorado de Gobierno de las Organizaciones del PAD-Escuela de Negocios de la misma universidad. Dirección electrónica: lizardo.vargas@udep.pe 


\title{
Videogaming: Attention Focalization and Reminiscence in Pre-Adolescents. Can They Focus on Simultaneous Processes and Give Space to Advertising?
}

\begin{abstract}
The purpose of this research is to identify, in pre-adolescents, the degree of effort or potential inhibition in the episodic and semantic evocation process that occurs when their attention is focalized due to their interaction with a videogame and to infer whether or not they can pay attention to and process advertising shown on videogames. A quantitative experiment was performed to analyze two groups of individuals belonging to the Formal Operations stage - cognitive development state proposed by Piaget and experienced by people of a certain age. Several effects on the reminiscence of episodic and semantic memories, in relation to time evocation and number of words used to express memories were observed. However, a significant difference in relation to the degree of certainty with which the evocation was exposed was not noticed. It was concluded that attention focalization by videogames does not demand different levels of cognitive efforts to process information in conjunction with semantic and episodic reminiscence, so advertising posted can really be taken care of.
\end{abstract}

Key words: Pre-adolescents; videogames; attention; memory; advertising. 


\section{Introducción}

Desde fines del siglo pasado los videojuegos empezaron a practicar el product placement, a través de la exhibición de marcas de diversos tipos en las pantallas de los usuarios, de ordinario con marcas ligadas con el tipo de juego, temática y audiencia, que mostraban diversos grados de efectividad (Glass, 2007).

Investigaciones muestran un alto y positivo nivel de recuerdo de los productos y las marcas, y con una mayor influencia en la actitud de los consumidores, cuando se práctica la exhibición de productos en los videojuegos en comparación con situaciones de publicidad convencional (Winkler, Buckner, 2006). Los autores citados llegan a la conclusión de que mostrar publicidad en los videojuegos puede funcionar eficazmente a favor de la construcción de marca, actitudes, y rememoración entre los jugadores de 8 a 18 años.

Los preadolescentes invierten una cantidad considerable de tiempo en la utilización de videojuegos. La iniciativa ACT (Act Against Violence, 2013) de la American Psychological Association señala que la media de horas semanales de un menor norteamericano entre los 8 y 18 años es de 13.2 de videjuego. Esta actividad da pie al desarrollo de una serie de destrezas, así como de formas de involucramiento cognitivo y emocional. Sin embargo, algunos investigadores han advertido que los videojuegos originan no solo un incremento subsecuente de problemas atencionales durante la infancia, sino también ocasionan una posible persistencia de ese problema en la adolescencia y hasta durante la adolescencia tardía (Swing, Gentiles, Anderson, Walsh, 2010).

Este estudio analiza el modo en que la memoria declarativa, es decir, aquella que puede ser expresada o comunicada por el individuo opera cuando un preadolescente focaliza la atención cuando se encuentra inmerso en el videojuego. Basado en la propuesta de Tulving (1972) de la división entre memoria semántica (de datos) y episódica (de eventos biográficos), se diseñó un experimento no paramétrico donde dos grupos de niños utilizaban un juego de vídeo y en un momento determinado se les hacía una serie de preguntas que exigían un esfuerzo de evocación en ambos tipos de memoria. Se espera observar una diferencia en la rememoración entre los dos tipos de memoria debido a que la episódica parece requerir más esfuerzo cognitivo mientras se procesa información en paralelo en comparación con la semántica (Surprenant y Neath, 2009; Baddeley, 2000; Styles, 2005).

Para analizar el proceso de evocación, se toman como marco teórico las propuestas de Tulving $(1972,2001)$ sobre la memoria y, por otro lado, las estructuras de procesamiento cognitivo propuestas por D. Kahneman (Kahneman y Frederick, 2002; Kahneman 2003), denominados Sistema 1 y Sistema 2, los cuales se presentan y explican en la revisión de la literatura.

Los niños seleccionados para este estudio se encuentran en la etapa de operaciones formales (11 años en adelante), de acuerdo con la categorización de desarrollo cognitivo de la psicología evolutiva clásica propuesta por Piaget (Piaget, 1981; Piaget; 1975; John, 1999). 
Se denomina Operaciones Formales a la etapa final de desarrollo cognitivo (Piaget, 1981; Piaget; 1975; Kramer, 2007). En ella se encuentran las personas de rangos etarios considerados como adolescencia y entrada a la edad adulta, y se caracteriza por su capacidad intelectual manifiesta en la utilización lógica de conceptos abstractos, así como la capacidad de ejercer pensamientos hipotéticos y deductivos, por ejemplo, en los posibles resultados y las consecuencias de las acciones, en contraste con las etapas previas del desarrollo cognitivo propuestas por el psicólogo, donde los niños tienden a pensar en términos concretos y específicos.

La revisión de la literatura revela que hay poca información sobre las operaciones de rememoración en los niños cuando se encuentran involucrados en alguna actividad (Belk, Mayer, Driscoll, (1984). Con el objeto de ampliar el cuerpo de conocimiento entre este grupo etario el presente artículo explora los efectos en la evocación en preadolecentes a la luz de la mencionada etapa de la teoría del desarrollo de Piaget. La actividad elegida para el estudio fueron los videojuegos, ya que se trata de un ejercicio con el que este grupo se encuentra familiarizado y reclama un alto nivel de foco atencional, lo cual tiene efectos sobre la dinámica cognitiva y de evocación de información (Simon, 1985), tanto semántica como episódica (Styles, 2005).

Este trabajo tiene por objeto examinar si existe una diferencia en la manera en que los preadolescentes recuerdan información de experiencias episódicas y semánticas, mientras interactúan con videojuegos. Cabe señalar que el efecto de los videojuegos sobre la memoria y la atención es tema de una extensa literatura, en particular, la relacionada con los desórdenes de déficit de atención e hiperactividad, pero también en pedagogía, dado que se comprende esta forma de entretenimiento como un instrumento de aprendizaje (Chan, Philip y Rabinowitz, 2006; Schmidt y Vandewater, 2008; Bloomberg, 1998). Otro campo de la literatura amplio versa sobre la violencia de los videojuegos y su efecto sobre las individuos (Anderson, 2004; Ferguson, 2011).

A lo largo del estudio se procura observar si existe una diferencia en la manera en que los preadolescentes pertenecientes al rango etario de operaciones formales recuerdan información de experiencias episódicas y semánticas mientras interactúan con videojuegos, mientras en simultáneo manejan información en los sistemas de procesamiento cognitivo denominados Sistema 1 y Sistema 2. Todo ello con el objeto de identificar la oportunidad de utilizar los videojuegos como un soporte publicitario válido en sí mismo y si puede convivir en paralelo con estímulos publicitarios del entorno.

\section{Revisión de la literatura: la memoria episódica y la semántica, y el procesamiento de información}

La presentación de la literatura académica cubre dos aspectos teóricos de este estudio. Por una parte, expone la propuesta de los modelos y las características de la memoria episódica y la semántica. En un segundo momento, presenta la teoría de procesamiento de información en paralelo, denominada como Sistema 1 y Sistema 2. 
La revisión de lo investigado, hasta el momento, expone los marcos teóricos en función de los cuales se han definido el diseño y los objetivos de este estudio, tal como se expuso en la introducción y se desarrollará con detalle en los acápites siguientes.

\section{La memoria episódica y la memoria semántica}

El psicólogo e investigador en neurociencia cognitiva -en particular sobre la memoria-, Endel Tulving, propone dos clases o géneros de operaciones, basado en la comprensión de la memoria como un sistema:

a) por una parte, la memoria episódica, vinculada con los acontecimientos experimentados personalmente de modo biográfico, como recordar un evento sucedido, el momento en que ocurrió y su contexto;

b) por otra parte, presentó la memoria semántica como aquella que versa sobre los hechos, e información general de la realidad y del mundo circundante del sujeto (Tulving, 1972, 2001; Squire y Zola, 1998).

Tulving señaló que su propuesta se fundaba en una teoría sistémica de la memoria, la que exigía, por lógica, que la memoria semántica fuera una consecuencia de la memoria episódica, pues un fenómeno vivido también es información sobre el mundo y la realidad -de ahí que considere ambas variantes como declarativas- (Tulving, 1984). Otros autores (Chao y Martin, 2001) opinan igual, sosteniendo que hay un grado de interdependencia entre la memoria episódica y la semántica, pues el conocimiento debe ser adquirido de forma temporal específica, así como sucede con todas las experiencias del individuo.

El desarrollo de ambos conceptos se realizó antes de la llegada de los instrumentos de neuroimagen, de forma que la explicación anatómica de los tipos de memoria episódica y semántica dependía de los estudios con sujetos que padecían lesiones o enfermedades cerebrales (Chao y Martin, 2001).

Por ejemplo, se observó que aquellos pacientes con un profundo deterioro de la memoria episódica pero con el conocimiento conservado de la semántica (como los amnésicos de Korsakoff, los cuales se caracterizan por síntomas de amnesia anterógrada -los nuevos acontecimientos no se guardan en la memoria a largo plazo- y retrógrada -incapacidad de recordar los eventos ocurridos antes del deterioro cerebral-), en contraste con otros pacientes con daño en la capacidad de la memoria semántica. Observado este efecto, Garrard, Perry y Hodges (1997) sostienen que los sistemas de memoria episódica y semántica se encuentran vinculados, pero solo parcialmente.

Con el uso de los equipos de neuroimagen, se lograron avances en la comprensión anatómica de los dos tipos de memoria propuestas por Tulving. Por ejemplo, se evidenció que las estructuras temporales mediales', en particular la formación del hipocampo,

1 El lóbulo temporal medial, que forma parte del lóbulo temporal del cerebro, es un sistema de estructuras anatómicamente relacionadas básicas para las funciones propias memoria declarativa episódica (el recuerdo consciente de hechos y eventos). 
son fundamentales para la codificación de los nuevos recuerdos episódicos. (Graham, Hodges, 1997; McClelland, McNaughton y O'Reilly, 1995). Ya es un acuerdo en la literatura neuropsicológica, que las estructuras del lóbulo temporal medio, incluido el hipocampo, son esenciales en la memoria declarativa (Tulving y Markowitsch, 1998).

Con el paso del tiempo, parece que esos recuerdos almacenados se independizan de su formación en el hipocampo y que la rememoración pasa a depender de los lóbulos frontales y el diencéfalo². Algunos estudios han evidenciado una participación de la corteza temporal inferolateral ${ }^{3}$ en la dinámica entre ambas (Garrard, Perry y Hodges, 1997).

No obstante, los datos neuroanatómicos aún son especulativos. Por ejemplo, cuando la memoria episódica se deteriora como resultado del lóbulo temporal medial, la memoria semántica debe ser correspondientemente reducida. Sin embargo, cuando la memoria episódica se deteriora, debido a una disfunción del lóbulo frontal, el aprendizaje de nueva información semántica debería ser posible. No obstante, esto no se observa en todos los casos (Janowskyet et. al., 1989; Schacter, 1987).

Una visión alternativa es que la memoria episódica no es totalmente crítica para la formación de recuerdos de naturaleza semántica. Esta opinión se basa en cierta evidencia de que los pacientes amnésicos pueden adquirir exitosamente un poco de conocimiento semántico luego de múltiples repeticiones (Shimamura y Squire, 1987; Hayman et al, 1993). Basándose en estos resultados, Tulving (1991) aseguró que la nueva información sí puede ingresar en la memoria semántica a través de los sistemas de percepción y de forma independiente al lóbulo temporal medial y otras estructuras diencefálicas dañadas en casos de amnesia.

El propio Tulving (2001) señala que la discusión sobre la existencia de la dimensión hipotética de la memoria episódica y semántica, enmarcada en el modelo sistémico -y no unitario- de la memoria (ver Surprenant y Neath, 2009; Baddeley, 2000) aún se mantiene en pie y que es necesario continuar con estudios posteriores para clarificar distintos aspectos y determinar la validez de las propuestas.

Estudios actuales en neurociencia también argumentan a favor de que ambos tipos de memoria no son del todo distintas, sino una distinción hipotética propia del modelo de Tulving (2001). En un estudio Squire y Zola (1998) anotaron que existen dos puntos de vista sobre cómo esta distinción puede verse reflejada en la organización de las funciones de la memoria en el cerebro: por una parte la memoria episódica y la memoria semántica son dependientes de la integridad del lóbulo temporal medial y estructuras de la línea media diencefálica. Este hecho predice que en los pacientes amnésicos con lóbulo temporal medial o daños diencefálico debe verse afectada proporcionalmente tanto en la memoria

2 El diencéfalo es una estructura ubicada en la parte interna central de los hemisferios cerebrales. Se encuentra entre los hemisferios y el tronco del cerebro, y a través de él pasan la mayoría de fibras que se dirigen hacia la corteza cerebral. Se encuentra compuesto por varias partes: tálamo, hipotálamo, subtálamo y epitálamo.

3 Estructura ubicada en la zona exterior e inferior del lóbulo temporal del cerebro. 
episódica como en la semántica. Por otro lado, en algunos casos de amnesia se observa que la memoria semántica está a salvo en contraste con la capacidad de la memoria episódica, con lo cual se puede pensar que los componentes episódicos y semánticos pueden ser disociados (Squire y Zola, 1998; Vargha-Khadem, et al., 1997).

Componentes existentes de la postura de Vargha-Khadem et al. (1997) incluyen la idea de que la adquisición de conocimiento de los hechos puede ocurrir independientemente de la memoria episódica, y la idea de que la amnesia anterógrada (cuando los nuevos acontecimientos no se guardan en la memoria a largo plazo) pueda observarse más grave en la memoria episódica que la memoria semántica. El autor y sus colegas señalan que el hipocampo es necesario para recordar experiencias de la vida actual (memoria episódica), pero no es necesario para la adquisición del conocimiento de los hechos (memoria semántica).

Al sostener la idea de que la adquisición de conocimiento de los hechos puede ocurrir independientemente de la memoria episódica, es muy posible que en los casos de amnesia anterógrada la memoria episódica sea más gravemente afectada que la memoria semántica. Tulving y Markowitsch (1998) están de acuerdo con ello y sugieren una comprensión de la memoria declarativa en función a las características y propiedades comunes a ambas, con lo cual se lograría una mayor precisión al modelo neuroanatómico propuesto por Vargha-Khadem et.al. (1997), en el que la memoria semántica depende de las regiones corticales del perihipocampo (zonas periféricas del hipocampo) ${ }^{4}$, mientras que la memoria episódica es dependiente del hipocampo.

En un estudio previo, Tulving et al. (1994) postulan una asimetría entre la actividad cortical en los casos de evocación episódica y semántica. El autor y colegas indican que los lóbulos prefrontales izquierdo y derecho son parte de una red neuronal extensa que atiende la evocación episódica, pero que estas dos zonas juegan diferentes papeles. La región cortical prefrontal izquierda se involucra en la recuperación de datos de la memoria semántica, y simultáneamente codifica nueva información; por otro lado, la región cortical prefrontal derecha se halla más involucrada en la recuperación de memorias de carácter episódico.

En otra investigación realizada por Butters et al. (1987) se pidió a personas con Alzheimer, sindrome de Huntington, y amnesia de Korsakoff realizar diversas tareas que implicaban ambas memorias, episódica y semántica. Los resultados del trabajo realizado por el equipo sugieren que las alteraciones como el síndrome del Alzheimer y pacientes con sindrome de Huntington reflejan diferentes procesos, los cuales afectan los procesos de la memoria episódica y de la semántica.

De este modo, los estudios realizados por los autores citados apuntan a que el esfuerzo cognitivo es distinto en los procesos de rememoración de los tipos de memoria, donde parece que la episódica requiere mayor esfuerzo cognitivo para ser declarada o manifestada.

4 Zona del encéfalo ubicada en el lóbulo temporal; se trata de una estructura cuyas funciones implican el aprendizaje y la memoria. 


\section{El procesamiento de información paralelo: Sistema 1 y Sistema 2}

Diversos autores han presentado desde diferentes perspectivas la dualidad con que los individuos procesan la información. La mayor parte de los trabajos observan el proce samiento como una capacidad psicológica superior, vinculada a las acciones como pensar, razonar y tomar decisiones. El consenso sobre la existencia y operación del procesamiento dual o paralelo es amplio en la literatura académica (Evans 2003).

El principal elemento común entre estas propuestas es el paralelismo del procesamiento cognitivo dual y el hecho de que cada ruta de procesamiento tenga características propias. La proposición más aceptada pertenece a los autores Stanovich y West (2000) quienes denominaron su modelo dual como Sistema 1 y Sistema 2. Esta compresión es compartida y complementada por Kahneman y Frederick (2002), y es el marco teórico de procesamiento cognitivo de este estudio.

Respecto al Sistema 1, este tiene unas características y funcionalidades reconocidas. El procesamiento de información por esta vía es generalmente rápido y automático, y no requiere mayor esfuerzo cognitivo; la elaboración mental es de carácter asociativo, y guiada por las costumbres de los sujetos, motivo por el cual se hace difícil de controlar o modificar (Kahneman, 2003). Debido a estas características, algunos autores optan por comprender el Sistema 1 como una organización de arquitectura procedimental 'implícita' (Wilson y Schooler, 2000; Evans, 2003).

Por otra parte, las funciones cognitivas del Sistema 2 son graduales y más pausadas en comparación con su contraparte. Tienen un carácter seriado y son secuenciales, razón por la cual requieren un mayor esfuerzo cognitivo y tienen más probabilidades de ser conscientemente monitoreadas y controladas de forma deliberada por el individuo, quien puede cumplir normas o reglas para modular el procesamiento en este sistema (Kahneman, 2003).

Distintos autores asocian el Sistema 2 con capacidades superiores como el lenguaje, la conciencia reflexiva, el control de ejecuciones de orden superior, y con la capacidad de pensar hipotéticamente sobre las posibilidades fácticas futuras (Reber 1993, Epstein E Pacini 1999, Stanovich 1999).

De acuerdo con las propiedades de cada sistema, en la literatura se ha determinado que el efecto concurrente de una tarea es el indicador más oportuno para identificar si un determinado proceso mental pertenece al Sistema 1 o al Sistema 2. Esto se debe a la limitación general de los procesos cognitivos: el esfuerzo psicológico para atender un proceso tiende a perturbar o interrumpir otro, mientras que aquellos procesos que no exigen tanto esfuerzo por parte del individuo no causan tanta interferencia cuando ocurren en simultáneo (Kahneman, 1973; Pashler, 1998).

Por otro lado, al analizar la relación entre los sistemas y la memoria es posible advertir que el Sistema 2 se comprende mejor dentro del marco del modelo unitario de la memoria de Baddeley y Hitch (1974), donde la información es procesada de forma secuencial en 
un espacio central de operaciones, mientras que el Sistema 1 es más independiente del mismo y más próximo a la memoria de corto plazo o activa.

Gilbert (2002) propuso que una de las acciones propias del Sistema 2 era la de supervisar la calidad de las operaciones mentales y la consecuente conducta del sujeto. El autor se basó en la hipótesis de que corresponde a la naturaleza de este sistema monitorear y modular el esfuerzo cognitivo que se requiere para mantener la atención simultánea y realizar operaciones que, de otra manera, podrían sufrir interferencia atencional (Gilbert 2002). Sin embargo, aún no hay un acuerdo en aspectos como este. Otros autores (Stanovich 2004, Wilson y Dunn 2004) plantean que atender una tarea de forma simultánea y automática no la convierte en una respuesta que se origina por debajo del nivel atencional, necesariamente capitalizado por el Sistema 2.

Esta investigación se basa en la perspectiva dual del procesamiento cognitivo propuesto por Kahneman y Frederick, similares a los de la teoría de la heurística-analítica de razonamiento. Los autores asumen que los procesos del Sistema 1 (heurístico) preceden intuitivamente a los juicios que deben ser aprobados por el Sistema 2 (analítico) que a menudo sucede por casualidad (Evans, 2006).

\section{Hipótesis}

Como se expuso en la introducción, este estudio tiene por objeto observar características en la evocación de recuerdos de naturaleza semántica y episódica cuando una población adolescente se encuentra en una situación de alto grado de focalización atencional, en concreto durante su interacción con videojuegos, pues como se explicó al inicio estos tienen el efecto de capitalizar el foco atencional de este grupo etario, lo cual tiene efectos sobre la dinámica cognitiva y de rememoración (Simon, 1985), tanto semántica como episódica (Styles, 2005).

Basado en los marcos teóricos presentados a lo largo de la revisión de la literatura, si el Sistema 2 requiere mayor esfuerzo cognitivo, conciencia reflexiva, control de ejecuciones de orden superior y la capacidad de proyectar posibilidades futuras (Kahneman, 2003; Reber 1993; Epstein y Pacini 1999; Stanovich 1999), la interacción con un videojuego capitaliza la atención del individuo. Esta actividad requiere por parte del sujeto un alto grado de procesamiento de información acorde con las características del descrito Sistema 2.

De acuerdo con lo expuesto en cuanto a los sistemas de procesamiento de información (Kahneman, 2003) y la naturaleza cognitiva de la evocación semántica y episódica, se propone que la rememoración de la primera es más cercana a la dinámica de procesamiento de información heurístico propio del Sistema 1, mientras la segunda se aproxima a un procesamiento de información más analítico, propio del Sistema 2 (Tulving, 1984; Chao, Martin, 2001).

En congruencia con ello, al involucrarse con el videojuego el individuo realiza y procesa información por el Sistema 2, con lo cual la rememoración semántica (Sistema 1) puede 
procesarse en simultáneo y originar una respuesta con mayor facilidad y fluidez que la rememoración episódica (la cual también exigiría el uso del Sistema 2, ya capitalizado por la atención requerida por el videojuego; Vargha-Khadem et al., 1997; Tulving y Markowitsch, 1998; Butters et al., 1987).

Lo expuesto puede graficarse de la siguiente manera (Gráfica 1)

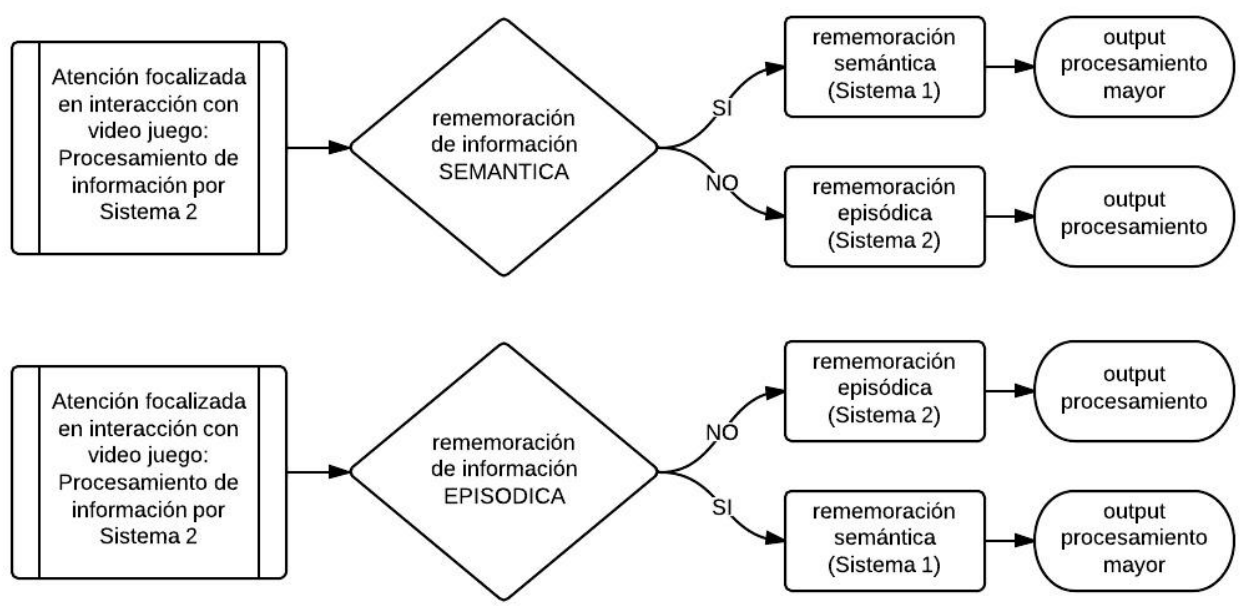

Gráfica 1. Flujo de procesamiento de información y sistema cognitivo utilizado para responder preguntas de tipo semántico o episódico.

Fuente: elaboración propia

De acuerdo con lo postulado en cuanto a la atención focalizada por el videojuego, los sistemas de procesamiento de información, y la naturaleza de los tipos de rememoración, se propone la siguiente hipótesis:

H1. La rememoración episódica requiere un mayor esfuerzo cognitivo en comparación con la rememoración semántica, cuando un sujeto perteneciente a la etapa de operaciones formales focaliza la atención interactuando con un videojuego.

\section{Metodología}

Para atender la hipótesis se realizó un estudio cuasiexperimental entre grupos, un grupo para observar la rememoración semántica y otro grupo para advertir la rememoración episódica. Se buscó una puntuación significativa en $p>.05$

Participantes. La población estudiada estuvo compuesta por una muestra no probabilística de $\mathrm{N}=28$, todos niños varones estudiantes de un colegio privado. La muestra se dividió en dos grupos (Grupo A, $n=14$; Grupo B, $n=14$ ). 
Todos los sujetos pertenecían al nivel socioeconómico medio-alto y alto. Fueron seleccionados de dos aulas distintas del colegio, de forma aleatoria simple. Todos cumplían con el requisito de encontrarse en el rango etario propio de la categoría de operaciones formales. Se obtuvo el consentimiento informado de los padres para seguir el protocolo de ética de la American Psychological Association. Las autoridades del colegio también conocieron el objetivo y procedimiento del estudio.

La estadística descriptiva etaria de los participantes de la muestra se exhibe en la tabla 1:

Tabla 1. Estadística descriptiva etaria de los grupos A y B

\begin{tabular}{|l|c|c|c|c|c|c|c|}
\hline & 11 años & 12 años & 13 años & Media & Mediana & Moda & DS \\
\hline Grupo A (episódico) & 1 & 11 & 2 & 12,1 & 12 & 12 & 0.47 \\
\hline $\begin{array}{l}\text { Grupo B } \\
\text { (semántico) }\end{array}$ & 0 & 13 & 1 & 12,1 & 12 & 12,1 & 0.26 \\
\hline
\end{tabular}

Variables. El diseño del experimento abarcó tres variables de medición que se aplicaron a ambos grupos por igual, y se determinaron porque manifestarían el nivel de exigencia en la rememoración tanto de tipo semántico como episódico. Las variables fueron: el lapso temporal que les tomaba rememorar y exponer la respuesta a cada pregunta (tr); el número de palabras con que los sujetos respondían a el conjunto de preguntas (np); y el grado de seguridad con el que exponían sus respuestas ( $g s$ ).

Procedimiento y materiales. Con el objeto de conseguir los datos, se diseñó y aplicó un cuestionario a ambos grupos de niños. El cuestionario constó de diez preguntas distintas para cada grupo. En el primer caso eran de carácter semántico (por ejemplo, 'cuál es la capital de Francia'), en el otro de carácter episódico (por ejemplo, 'qué comiste el sábado pasado por la noche').

Para registrar las respuestas se elaboró una ficha impresa que el experimentador completó conforme entrevistaba a cada sujeto. En la ficha, fueron registrados los siguientes datos: la edad del niño y las variables $t r, n p, g s$. Para registrar tr se utilizó un cronómetro, y para sg se determinó una escala semántica de tres puntos (seguridad alta, seguridad normal, y seguridad baja) que era completada a criterio del experimentador, en función de la certeza verbal y no verbal percibida en cada respuesta de los individuos.

El cuestionario se realizó de forma individual y oral, en una sala dentro del colegio donde el infante se sentó en un sofá frente a la pantalla del televisor para jugar el videojuego en un ambiente similar a como lo haría en su hogar, en el de un pariente o en el de un amigo. Los investigadores (principal y asistente) se sentaron alineados con él, a su lado derecho.

Antes de iniciar el experimento, el entrevistador utilizó, como señuelo, que se trataba de estudiar el modo como los niños de su edad interactuaban con los videojuegos 
y atendían otras actividades mientras jugaban. Se les explicó que jugarían un videojuego de Play Station 3 (ps3) durante 10 minutos y que, en un momento determinado, se le plantearían unas preguntas a las que debían responder espontáneamente mientras continuaba jugando.

El videojuego era de carácter bélico, no requería destrezas estratégicas y era conocido por los participantes. Si perdían durante el juego, podían reiniciar la actividad desde el punto donde se habían detenido hasta cumplir los diez minutos. El investigador no ofreció asistencia al entrevistado durante el juego.

Todos los participantes jugaron el mismo juego, en el mismo nivel y grado de dificultad. Durante los primeros 3 minutos no se les interrumpió, con el objeto de que se iniciara el proceso de involucramiento atencional en el videojuego. En el minuto 3 se procedió a hacerles las preguntas (episódicas o semánticas, de acuerdo con cada grupo) y a registrar las respuestas, hasta llegar al minuto 10 en que se les pedía que pausaran el juego.

\section{Resultados}

Luego de procesar los datos obtenidos de la experimentación se advirtió que los grupos no tenían características paramétricas: los datos no tuvieron una distribución normal, y los grados de varianza en dos de las variables fue alta ( $t r=1,81 ; n p=2,75$ ), y baja en el caso del índice de seguridad en las respuestas ( $s g=0,133)$. Debido a estas características, se optó por utilizar una prueba de la $t$ de Mann-Whitney para realizar un análisis comparativo de medias de las variables tr y $n p$. En el caso de la variable sg, al tener una varianza menor, se consideró oportuno utilizar la prueba de la t de Welch. Los resultados se presentan en los párrafos a continuación.

En cuanto al tiempo de respuesta (tr) que los sujetos requirieron para rememorar las preguntas de naturaleza episódica o bien semántica, la prueba de Mann-Whitney mostró una diferencia significativa: $z=-2,138, \mathrm{n}_{\mathrm{A}}=14, \mathrm{n}_{\mathrm{B}}=14, p=.033$, prueba bilateral.

Por otro lado, la prueba de Mann-Whitney sí evidenció una diferencia significativa en la cantidad de palabras ( $n p$ ) con que los sujetos respondieron a las preguntas de naturaleza episódica o semántica: $z=-4,461, \mathrm{n}_{\mathrm{A}}=14, \mathrm{n}_{\mathrm{B}}=14, p=.000$, prueba bilateral.

Finalmente, en cuanto al nivel de seguridad (sg) con que los sujetos respondieron a las preguntas que requerían un esfuerzo de rememoración episódico o semántico, la estadística no evidenció una diferencia significativa: Welch $t(26)=0,133, p=0,880$, prueba bilateral.

\section{Conclusiones y discusión posterior}

La hipótesis del estudio estipulaba que en situaciones de atención focalizada por un videojuego, los niños debían hacer un mayor esfuerzo en la evocación de información episódica comparado con la semántica. Así, la investigación observó la relación entre las tres variables medidas: el nivel de seguridad en la respuesta a lo preguntado a los 
participantes, lo que tardaban en responder y el número de palabras con que verbalizaban la rememoración en cada caso. De este modo, el resultado congruente era observar un tiempo de respuesta menor en la evocación semántica, mayor seguridad en sus respuestas y un menor número de palabras para verbalizarlas.

En cuanto al tiempo de respuesta (tr), los resultados favorecieron la hipótesis alternativa al indicar que tr para evocar información de tipo episódico era menor que el semántico. Respecto al número de palabras (np) utilizadas por los sujetos para responder las preguntas, los datos obtenidos evidenciaron que el esfuerzo para la rememoración episódica requiere más palabras que en el de tipo semántico. Este resultado también favorece la hipótesis alternativa.

Sin embargo, en cuanto al grado seguridad (gs) reportado, no se evidenció diferencia en el esfuerzo cognitivo de rememoración para exponer las respuestas de naturaleza semántica o episódica, con lo cual en este aspecto se cumple la hipótesis nula.

A partir de los resultados obtenidos es posible señalar que, en la focalización atencional originada por la interacción con videojuegos, el grado de procesamiento de información en paralelo que realizan los niños a través del Sistema 1, requiere menos esfuerzo para traer a colación datos episódicos, en comparación con el esfuerzo necesario para rememorar información de naturaleza semántica.

Una explicación de ello puede ser que la información semántica necesite de menos cues (guías) para evocar la información, en contraste con los datos de orden episódico, los cuales parecen ser más articulados o dependientes de cues adicionales (Surprenant y Neath, 2009). De este modo, la menor articulación de los datos semánticos le permite compartir el buffer atencional y de procesamiento de información y operar en los Sistemas 1 y 2 en simultáneo (Styles, 2005).

La figura 1 expuesta antes es congruente con lo propuesto en la hipótesis, donde se postula que en situaciones de atención focalizada por un videojuego, los niños debían hacer un mayor esfuerzo en la evocación de información episódica comparado con la semántica (dando por supuesto que la información semántica requiere procesamiento por la el sistema 1, y la episódica, por el 2) (Kahneman y Frederick, 2002). Las hipótesis alternativas sustentan este argumento.

No obstante, a pesar de que el grado de certidumbre en la respuestas evocadas (sg) con que los sujetos de control y experimental exponían sus recuerdos no manifestó una distinción estadística significativa, los resultados sí indican procesamiento en simultáneo por ambos sistemas, aunque de acuerdo con cada tipo de información procesada, cuando la atención es focalizada con el videojuego.

Parece que entre los niños estudiados ni la focalización atencional ni el procesamiento en paralelo durante la interacción con el juego pareció inhibir su conocimiento percibido necesario de expresar la respuesta que ellos consideraban adecuada (Moorman, Diehl, et. al., 2004). Es plausible sostener que mientras interactúan con el videojuego, el conjunto 
de preadolescentes analizado procesa la información de forma selectiva y a niveles de relevancia por debajo del estímulo principal de focalización.

Cabe también la posibilidad de que sea un efecto originado por la ausencia de riesgo en caso de que las respuestas fueran en alguna forma erradas, y que por ello no perciban contingencia alguna. Convienen estudios posteriores para analizar este fenómeno.

Siendo el caso, al nivel que permite esta investigación puede afirmarse que mientras un niño interactúa con un videojuego sí puede realizar procesos paralelos de evocación, en particular, de tipo semántico. En cierto modo queda de manifiesto que la memoria se encuentra activa, con lo cual es viable concluir que estímulos como los publicitarios - o de distinta naturaleza- sí pueden ser codificados y procesados y almacenados, con un efecto positivo en la construcción de marca cuando, por ejemplo, la publicidad es exhibida como un elemento visual dentro del videojuego (product placement) o mientras se atiende en simultáneo, lo cual es congruente con la ampliamente aceptada teoría de la atenuación del filtro atencional de A. Treisman (Treisman, Kahneman y Burkell, 1983; Treisman y Sato, 1990), la cual postula que los estímulos del entorno sí son atendidos y procesados pero con diversos grados de involucramiento de acuerdo con la relevancia que el sujeto les otorgue.

De acuerdo con lo observado, se puede identificar la oportunidad de utilizar los videojuegos como plataformas válidas para su uso en estrategias de marca en las comunicaciones de marketing, y que a su vez estos pueden convivir en simultáneo con estímulos publicitarios del entorno cumpliendo adecuadamente diversos objetivos de comunicación comercial.

\section{Bibliografía}

ACT Iniciative: Act Against Violence, American Psychological Association (2013). www.actagainstviolence.org. Recuperada: el 10 de julio de 2013. Disponible en: http://www.education.com/facts/ quickfacts-video-games/average-amount-playing/

Martin, A. y Chao, L. (2001). Semantic memory and the brain: structure and processes. Current Opinion in Neurobiology, 11(2), 194-201.

Craig, A. (2004). An update on the effects of playing violent video games. Journal of Adolescence, 27, $113-122$.

Baddeley, A., y Hitch, G. (1974). Working memory. In The Psychology of Learning and Motivation, 8, ed. Bower GA, pp. 47-90. New York: Academic Press.

Baddeley, A. (2000). The episodic buffer: a new component of working memory? Trends in cognitive science. 4, 417-423.

Belk, R., Mayer, R., y Driscoll, A. (1984). Children's recognition of consumption symbolism in children's products. Journal of Consumer Research, 386-397.

Butters, N., Granholm, E., Salmon, D. P., Grant, I., y Wolfe, J. (1987). Episodic and semantic memory: A comparison of amnesic and demented patients. Journal of Clinical and Experimental Neuropsychology, 9(5), 479-497. 
Chan, A. y Rabinowitz, T. (2006). A cross-sectional analysis of video games and attention deficit hyperactivity disorder symptoms in adolescents. Annals of General Psychiatry, 5, 16-11.

Chen, S. y Chaiken, S. (1999). The heuristic-systematic model in its broader context. In Dual-process theories in social psychology, ed. Chaiken S, Trope Y, pp. 73-96. New York: The Guildford Press.

Coates, S., Butler, L. y Berry, D. (2004). Implicit memory: a prime example for brand considerations and choice. Applied Cognitive Psychology, 18, 1195-1211.

Swing, E., Gentile, D.; Craig A. y Walsh, D. (2010), Television and Video Game Exposure and the Development of Attention Problems. Pediatrics, 126(214), 214-221.

Epstein, S y Pacini, R. (1999). Some basic issues regarding dual-process theories from the perspective of cognitive-experiential theory. In Dual-process theories in social psychology, ed. Chaiken, S., Trope, Y., 462-482. New York: The Guildford Press.

Evans, J. (2003), In two minds: Dual process accounts of reasoning. Trends in Cognitive Science, 7, 454-459.

Ferguson, C. (2011). Video Games and Youth Violence: A Prospective Analysis in Adolescents. Journal of Youth E Adolescence, 40(4), 377-391.

Gilbert, D. (2002). Inferential correction. In T. Gilovich, D. Griffin, E D. Kahneman (Eds.), Heuristics and biases (167-184). New York: Cambridge University Press.

Glass, Z. (2007). The effectiveness of product placement in video games. Journal of Interactive Advertising, 8(1), 1-27.

Graham, K. y Hodges, J. (1997). Differentiating the roles of the hippocampal complex and the neocortex in long-term memory storage: evidence from the study of semantic dementia and Alzheimer's disease. Neuropsychology, 11, 77-89.

Hayman C., MacDonald, C. A. y Tulving, E. (1993). The role of repetition and associative interference in new semantic learning in amnesia: A case experiment. Journal of Cognitive Neuroscience, 5, 375-389.

Jacoby, L. (1983). Perceptual Enhancement: Persistent Effects of an Experience. Journal of Experimental Psychology: Learning, Memory, and Cognition, 9(1), 21-38.

Janowsky J., Shimamura A. y Squire, L. (1989). Source memory impairment in patients with frontal lobe lesions. Neuropsychologia, 27, 1043-1056.

Kahneman, D. y Frederick, S. (2002). Representativeness revisited: Attribute substitution in intuitive judgement. In Heuristics and biases: The psychology of intuitive judgment, ed. Gilovich, T., Griffin, D., Kahneman, D., 49-81. Cambridge: Cambridge University Press.

Kahneman, D. (2003). A Perspective on Judgment and Choice. American Psychologist, 58(9), 697-720.

Kahneman, D. (1973). Attention and effort. Englewood Cliffs, NJ: Prentice-Hall.

Kahneman, D. y Thaler, R. (2006) .Utility Maximiaztion and Experienced Utility. Journal of Economic Perspectives, 20(1), 221-234.

Kramer, J. (2007). Is abstraction the key to computing?. Communications of the ACM, 50(4), 36-42.

Squire, L. y Zola, S. (1998). Episodic Memory, Semantic Memory, and Amnesia. Hippocampus, 8, 205-211.

McClelland, J., McNaughton, B. y O'Reilly, R. (1985). Why are there comple- mentary learning systems in the hippocampus and neocortex? Insights from the successes and failures of connectionist models of learning and memory. Psychological Review, 102, 419-57. 
Moorman, C., Diehl, K., Brinberg, D. y Kidwell, B. (2004). Subjective Knowledge, Search Locations, and Consumer Choice. Journal of Consumer Research, 31, 673-680.

Novemsky, N. y Kahneman, D. (2005). The boundaries of loss aversion. Journal of Marketing Research, 42, 119-128.

Garrard, P., Perry, R. y Hodges, J. (1997). Disorders of semantic memory. Journal of Neurology, Neurosurgery, and Psychiatry; 62, 431-435.

Piaget, J. (1975). Problemas de psicología genética. Barcelona: Ariel.

Piaget, J. (1981). Seis estudios de psicología. 11. ${ }^{\text {a }}$ edición. Barcelona: Seix Barral.

Reber, S. (1993). Implicit Learning and Tacit Knowledge. Oxford: Oxford University Press.

Schacter, D. (1987). Memory, amnesia, and frontal lobe dysfunction. Psychobiology, 15, 21-36.

Schmidt, M. y Vandewater, E. (2008) Children and Electronic Media. The Future of Children, 18(1), 63-85.

Shimamura, A. y Squire, L. (1987). A neuropsychological study of fact memory and source amnesia. Journal of Experimental Psychology [Learning Memory and Cognition], 13, 464-473.

Simon, T. (1985). Play and learning with computers. Early Child Development and Care, 19(1-2), 69-78.

Squire, L. R., y Zola, S. M. (1998). Episodic memory, semantic memory, and amnesia. Hippocampus, 8(3), 205-211.

Stanovich, K. (2004). The robot's rebellion: Finding meaning the age of Darwin. Chicago: Chicago University Press.

Stanovich, K. (1999). Who is Rational? Studies of Individual Differences in Reasoning. Mahway, NJ: Lawrence Elrbaum Associates.

Stanovich, K. y West, R. (2000). Individual differences in reasoning: Implications for the rationality debate. Behavioral and Brain Sciences, 23, 645-665.

Styles, E. (2005). Attention, perception and memory: An integrated introduction. Psychology Press.

Surprenant, A. y Neath, I. (2009). Principles of memory, New York: Psychology Press.

Treisman, A., Kahneman, D., y Burkell, J. (1983). Perceptual objects and the cost of filtering. Perception E Psychophysics, 33(6), 527-532.

Treisman, A., y Sato, S. (1990). Conjunction search revisited. Journal of Experimental Psychology: Human Perception and Performance, 16(3), 459.

Tulving, E. (1972). Episodic and semantic memory. In: Tulving E., y Donaldson W., eds. Organisation of memory. New York: Academic Press.

Tulving, E. (1984). Relations among components and processes of memory. Behavioral and Brain Sciences, 7, 257-68.

Tulving, E., Kapur, S., Craik, F. I., Moscovitch, M., y Houle, S. (1994). Hemispheric encoding/retrieval asymmetry in episodic memory: positron emission tomography findings. Proceedings of the National Academy of Sciences, 91(6), 2016-2020.

Tulving, E., y Markowitsch, H. J. (1998). Episodic and declarative memory: role of the hippocampus. Hippocampus, 8(3), 198-204. 
Tulving, E. (2001). Episodic memory and common sense: how far part? Philosophical Transactions B, The Royal Society, 356, 1505-1515.

Tulving, E. (2002). Episodic memory: from mind to brain. Annual Review of Psychololy, 53, 1-25.

Vargha-Khadem, F., Gadian, D. G., Watkins, K. E., Connelly, A., Van Paesschen, W., y Mishkin, M. (1997). Differential effects of early hippocampal pathology on episodic and semantic memory. Science, 277(5324), 376-380.

Ward, A. (2004). Attention. A Neuropsychological approach. East Sussex: Psychology Press

Wilson, T. y Dunn, E. (2004). Self-knowledge: Its limits, value and potential for improvement. Annual Review of Psychology, 55(4), 93-518.

Wilson, T., Lindsey, S. y Schooler, T. (2000). A Model of Dual Attitudes. Psychological Review, 107(1), 101-26.

Winkler, T., y Buckner, K. (2006). Receptiveness of gamers to embedded brand messages in advergames: Attitudes towards product placement. Journal of Interactive Advertising, 7(1), 37-46. 